\title{
Psychosocial Implications of Prophylactic Bilateral Mastectomy
}

\author{
H.M. Bebbington and L.J. Fallowfield \\ CRC Psychosocial Oncology Group, \\ Department of Oncology, University College \\ London, London, UK
}

Introduction: The removal of healthy breast tissue to prevent the development of breast cancer is seen by many to be a radical surgical intervention. As the numbers of women contemplating such surgery are increasing it is essential that the psychological costs and benefits of prophylactic bilateral mastectomy are established.

Aims: This prospective study aims to measure psychological morbidity, to describe the decision-making process and to determine communication and counselling needs in women who both opt for and decline prophylactic bilateral mastectomy. It also aims to identify preoperative factors that may predict post-operative distress in women who opt for this surgery.

Method: The objective is to recruit 152 women who have a strong family history of breast cancer. This total will comprise two groups of 76, one group opting for surgery the other declining. To date the study has recruited 78 women who have opted for surgery and 63 who have declined. Women are interviewed before surgery, then at 6 and 18 months postoperatively. Those declining surgery are interviewed after first contact, then 18 months later. All women are given standard psychological questionnaires to complete at each interview.

Results: Interim analysis shows that women opting for surgery have a more accurate perception of the general population risk, but when it comes to personal risk estimates, a much higher percentage of the surgical group felt it was inevitable that they would develop the disease. Analysis of the General Health Questionnaire, a screening tool for psychiatric distress, has shown that women opting for surgery are also more distressed than those opting for regular surveillance.

Discussion: A strong factor that appears to be associated with those opting for surgery, and which also appears to influence decision making, is personal family history and experience of breast cancer within the family. Other issues that are emerging from the interviews include: the feelings of isolation being experienced by those women opting for this intervention; a lack of information about prophylactic mastectomy and reconstruction issues for those who have never had breast cancer; the inability to get funding for the operation in some areas of the UK and postoperative complications. 


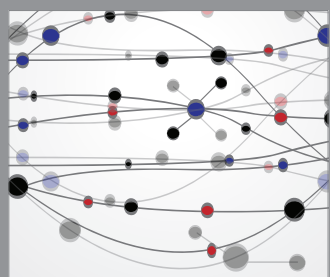

The Scientific World Journal
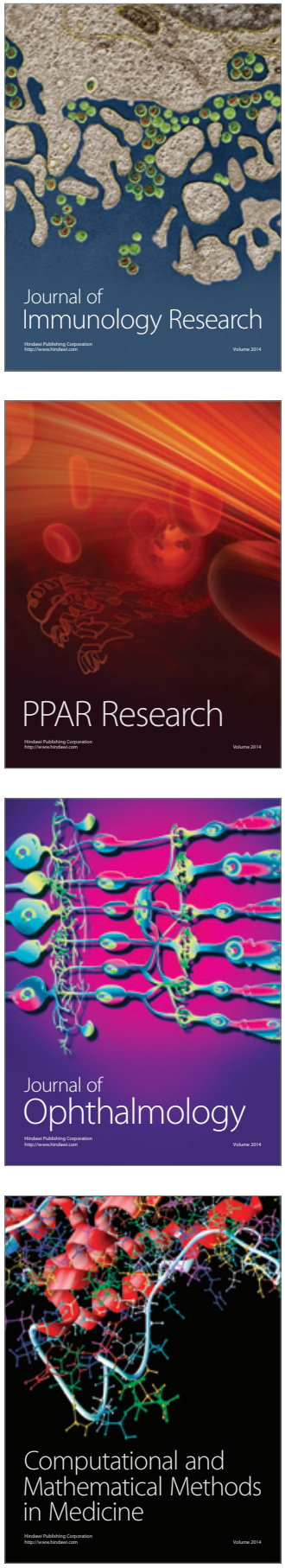

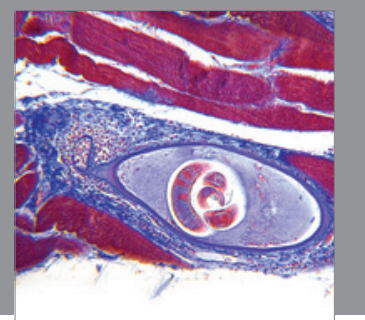

Gastroenterology

Research and Practice
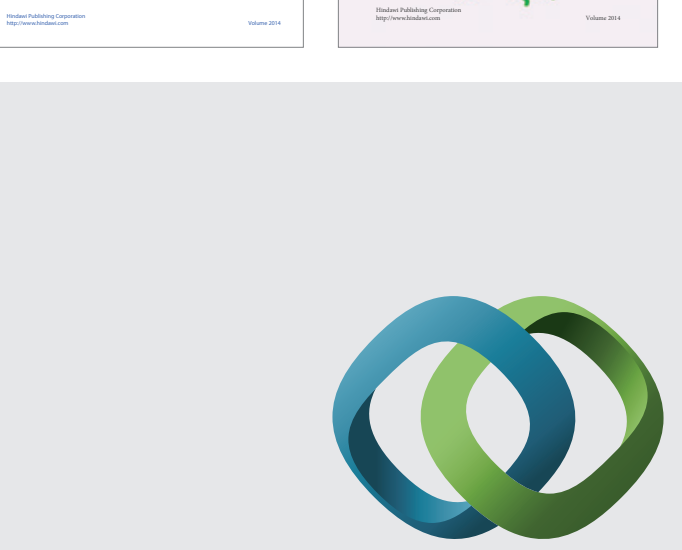

\section{Hindawi}

Submit your manuscripts at

http://www.hindawi.com
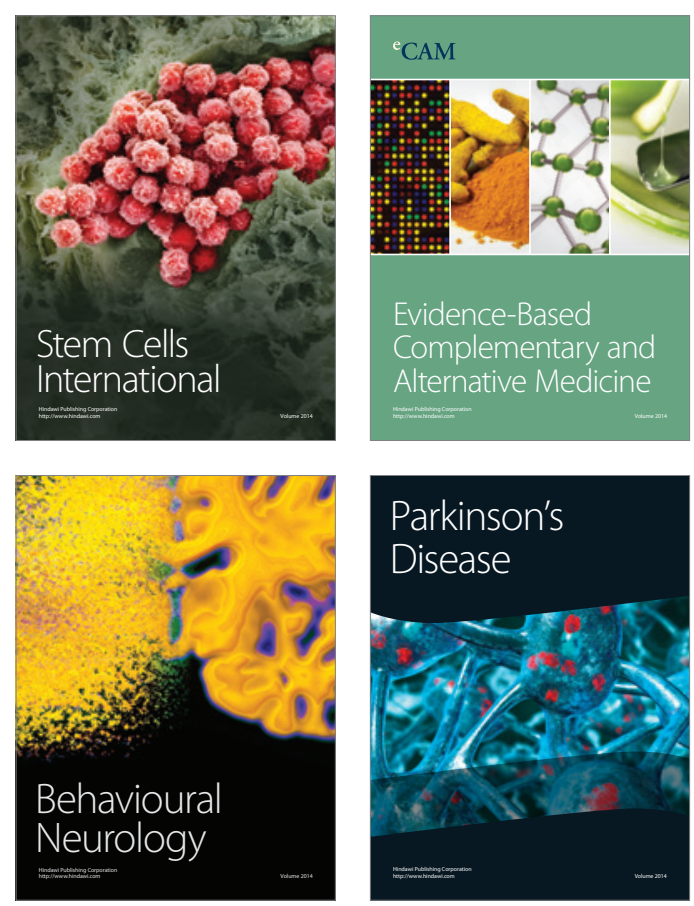

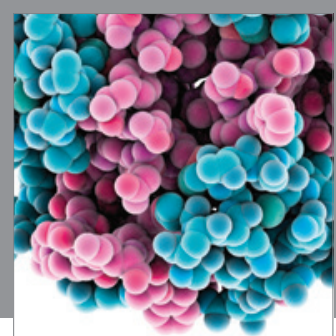

Journal of
Diabetes Research

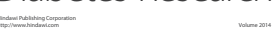

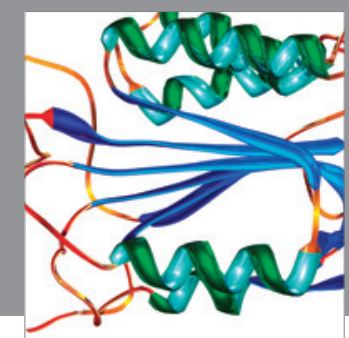

Disease Markers
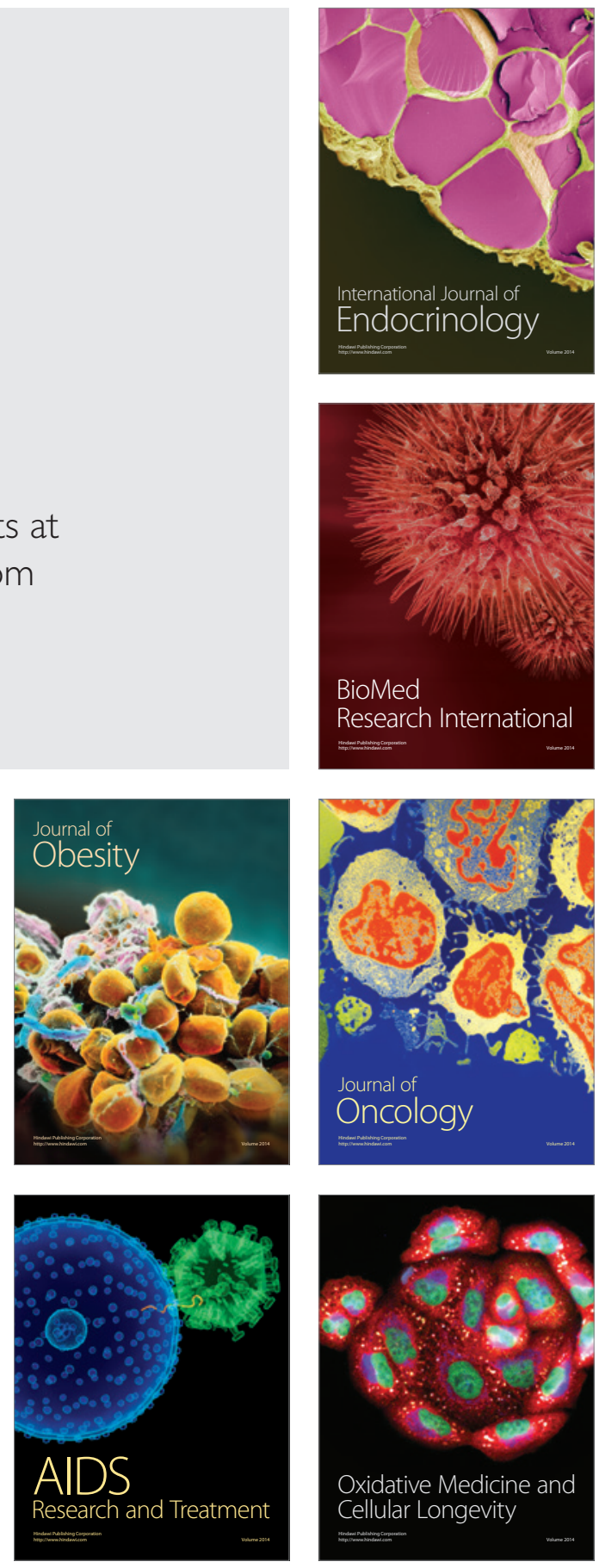